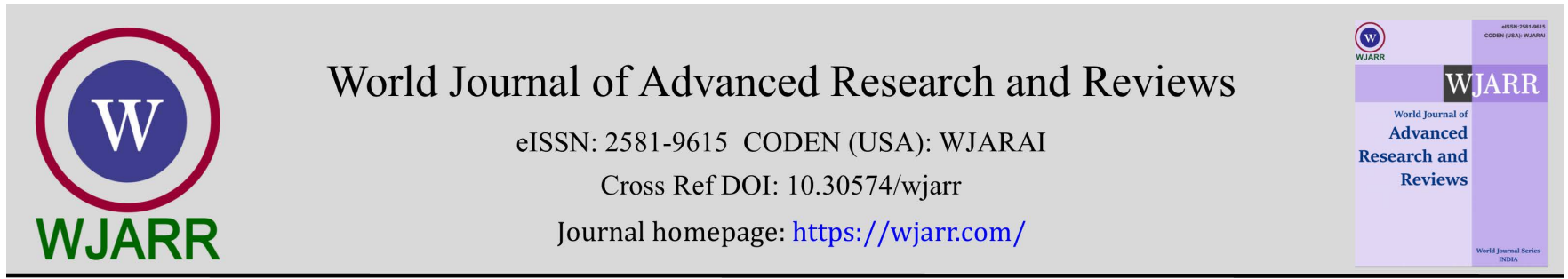

(RESEARCH ARTicle)

Check for updates

\title{
Analysis of gene expression profiles of Enterococcus faecalis induced by type I collagen
}

\author{
Yoki Hirakawa, Sadaomi Sugimoto, Norimasa Tsuji, Takeshi Inamoto and Hiroshi Maeda * \\ Department of Endodontics, Osaka Dental University 8-1 Kuzuhahanazonocho Hirakata, Osaka 573-1121, JAPAN.
}

World Journal of Advanced Research and Reviews, 2022, 13(01), 129-139

Publication history: Received on 23 November 2021; revised on 26 December 2021; accepted on 28 December 2021

Article DOI: https://doi.org/10.30574/wjarr.2022.13.1.0742

\begin{abstract}
Enterococcus faecalis is an etiological agent of endodontic infections. The present study was performed to investigate the gene profiles of E. faecalis induced by type I collagen stimulation. E. faecalis ATCC 19433 was cultivated with [collagen $(+)$ ] or without type I collagen [collagen $(-)$ ], and transcriptome analysis was performed using highthroughput sequencing technology. A total of $3.6 \mathrm{gb}$ of information was obtained by sequence analysis and 77 differentially expressed genes (DEGs) between the two culture conditions were identified. Among the 77 DEGs, 35 genes were upregulated in collagen (+) E. faecalis, whereas 42 genes were downregulated. Gene Ontology (GO) enrichment analysis was performed and 11 GO terms, including metalloendopeptidase activity (G0:0004222) and two related G0 terms (G0:0031012, G0:0044421), were significantly enriched in the set of upregulated genes. We focused on an upregulated DEG belonging to the matrixin metalloprotease gene family, and matrix metalloprotease (MMP) activities of the bacterial cell were examined. The generic MMP, MMP-8, and MMP-9 activities of collagen (+) E. faecalis were significantly higher than those of collagen ( - E. faecalis. These results suggested that contact with type I collagen may alter the gene expression profile of E. faecalis, and upregulation of metalloprotease genes may result in enhanced MMP activities in E. faecalis.
\end{abstract}

Keywords: Enterococcus faecalis; Transcriptome analysis; Matrix metalloprotease; Gene ontology (GO) enrichment analysis

\section{Introduction}

Enterococcus faecalis is a facultative anaerobic gram-positive bacterium that is known to be a commensal organism in the gastrointestinal tract and oral cavity. However, this bacterium has also been shown to act as a pathogen in serious systemic infections, including bacteremia, endocarditis, urinary tract infections, surgical wound infections, and intraabdominal and intrapelvic infections, especially in immunocompromised hosts [1]. E. faecalis is also known to be one of the major causes of serious nosocomial infections. The spread of vancomycin-resistant enterococci (VRE) is a universal problem in hospitals and long-term care facilities [2].

In the oral cavity, E. faecalis has been detected in root canal infections [3] and infected dentin [4]. In endodontic infections, E. faecalis is one of the most frequently implicated pathogens in both primary and secondary root canal infections [5]. As E. faecalis has been frequently isolated from the obturated root canals of teeth with apical periodontitis, this bacterium has been suggested to have a pathogenic role in endodontic treatment failure [6]. It has been reported that E. faecalis shows resistance to root canal treatment, including the medicaments used [7]. It has also been reported that $E$. faecalis can survive in root canals without the support of other symbiotic bacteria [8]. Due to these characteristics, E. faecalis is the most widely implicated pathogen in persistent root canal infections. For the host, especially under conditions of immune compromise, root canals connected to alveolar bone could represent a route for

\footnotetext{
${ }^{*}$ Corresponding author: Hiroshi Maeda

Department of Endodontics, Osaka Dental University 8-1 Kuzuhahanazonocho Hirakata, Osaka 573-1121, JAPAN.

Copyright $(2022$ Author(s) retain the copyright of this article. This article is published under the terms of the Creative Commons Attribution Liscense 4.0.
} 
oral bacteria associated with risks of morbidity and mortality. In this regard, E. faecalis, a persistent invader of the root canal, is one of the most high-risk pathogens.

In addition to antibiotic resistance, a series of virulence factors of $E$. faecalis have been reported. The surface protein is involved in immune evasion [9]. Cytolysin plays a role in hemolytic and bactericidal activity against other gram-positive bacteria [1]. Aggregation substances, gelatinase, collagen binding protein, etc., have also been reported to be involved in the pathogenesis of various infectious diseases [1]. Collagen-binding protein and protease have been reported to be virulence factors in endodontic infection [10]. E. faecalis has been reported to play critical pathogenic roles in both systemic and dentinal infections, and collagen is the common host component between these two types of infectious lesions. We hypothesized that E. faecalis may have common virulence factors against collagen between systemic and dentin infection. Contact with type I collagen may alter the mRNA profile of E. faecalis and lead to adaptation to the root canal environment and enhancement of virulence. In the present study, transcriptome analysis was performed to identify type I collagen induced genes. Further, based on the results of transcriptome analysis, the phenotypic alterations to matrix metalloprotease (MMP) activity attributable to contact with type I collagen were examined.

\section{Material and methods}

\subsection{Bacterial strain and culture conditions}

E. faecalis ATCC 19433 was cultivated in brain-heart infusion (BHI) broth (Nissui, Tokyo, Japan) at $37^{\circ} \mathrm{C}$ with $(1 \mu \mathrm{g} / \mathrm{mL})$ or without type I collagen (Nitta Gelatin, Osaka, Japan). The optical density at $660 \mathrm{~nm}$ (OD 660) of the medium was monitored using a MiniPhoto absorbance meter (Taitec, Koshigaya, Japan).

\subsection{Transcriptome analysis}

E. faecalis was harvested at mid-exponential phase, and total RNA was extracted using a RiboPure ${ }^{\mathrm{TM}}$ RNA Purification Kit (Thermo Fisher Scientific, Waltham, MA, USA). Transcriptome analysis was performed by Rhelixa (Tokyo, Japan). Briefly, 16S and 23S rRNA were removed from the total RNA using a Ribo-Zero Plus rRNA Depletion Kit (Illumina, San Diego, CA, USA), and a strand-specific library was constructed by cDNA synthesis and adaptor ligation using NEBNext an Ultra RNA Library Prep Kit for Illumina (Illumina). Sequencing was performed using an Illumina NovaSeq 6000 (Illumina). Quality check and trimming bad quality reads and adaptor sequence were performed using FastQC software version 11.7 (Babraham Instituite: https://www.bioinformatics.babraham.ac.uk/projects/fastqc/) and Trimmomatic version 0.38 (Illumina), respectively. The trimmed sequence reads were mapped to the reference genome sequence of E. faecalis ATCC 19433 on the website of the National Center for Biotechnology Information (https://www.ncbi.nlm.nih.gov/genome/browse\#!/prokaryotes/enterococcus) using HISAT2 version 2.1.0 (11).

The expression level of each gene was evaluated as transcripts per million (TPM) calculated with featureCounts version 1.6.3 (SOURCEFORGE SUBREAD: https://sourceforge.net/projects/subread/files/subread-1.5.2/). Differentially expressed genes (DEGs) between the samples were detected using edgeR software version 3.26.8 (Bioconductor: https://bioconductor.org/packages/release/bioc/html/edgeR.html). Transcripts showing a 2 -fold increase or reduction to $1 / 2$ or less in expression with $P<0.05$ were considered as DEGs, and a volcano plot was made using plotly version 4.9.2.1. (Plotly Python Open-Source Graphing Library: https://plotly.com/python/).

Gene Ontology (GO) enrichment analysis of a subset of DEGs and creation of an oriented graph were conducted with TopGO ${ }^{3}$ version 2.36.0 (Bioconductor: https://bioconductor.org/packages/release/bioc/html/topGO.html). GO terms (MF, molecular function; BP, biological process; CC, cellular component) with $P<0.05$ (Fisher's exact test) were distinguished in this study.

\subsection{MMP activity assay}

The MMP activities of the whole-cell lysate of $E$. faecalis were assessed using a SensoLyte ${ }^{\circ} 520$ (Generic MMP, MMP-1, $2,8,9)$ Activity Kit*Fluorimetric* (AnaSpec, San Jose, CA, USA) according to the manufacturer's instructions. Briefly, 50 $\mu \mathrm{L}$ of the sonicated bacterial cell suspension in assay buffer $(3 \mathrm{mg} / \mathrm{mL})$ was mixed with $50 \mu \mathrm{L}$ of MMP substrate solution provided with the kit. The enzyme reaction was carried out on 96-well plates (Brand GmbH + Co. KG, Wertheim, Germany) and the fluorescence intensity was measured at $490 \mathrm{~nm} / 520 \mathrm{~nm}$ and recorded every $5 \mathrm{~min}$ for $8 \mathrm{~h}$ using a SpectraMax M5 Multi-Mode Microplate Reader (Molecular Devices Co., San Jose, CA, USA). The fluorescence intensity was normalized relative to assay buffer with the substrate and shown in relative fluorescence units (RFU). Recombinant human MMP-1, -2, -8, and -9 (0.5 units/mL; Life Laboratory Co., Sendai, Japan) were used as controls at final concentrations of $0.25 \mathrm{unit} / \mathrm{mL}$. Triplicate measurements were performed twice. Mean RFU at the measurement time 
of 210 min were compared between the samples by the Mann-Whitney U test. In all analyses, $P<0.05$ was taken to indicate statistical significance.

\section{Results}

\subsection{Sequencing}

Totals of 20173020 and 16078348 reads were obtained from E. faecalis cultivated with type I collagen [collagen(+)] and without collagen [collagen $(-)]$, respectively. The reads were mapped to the E. faecalis ATCC 19433 genome sequence (NCBI Reference Sequence: NZ_ASDA00000000.1), and 99.10\% [collagen(+)] and 99.11\% [collagen(-)] of the reads were matched to the annotated coding sequences.

\subsection{DEG and GO enrichment analysis}

A total of 77 genes were found to be differentially regulated in collagen(+) versus collagen(-) E. faecalis (Fig. 1). Thirtyfive genes were upregulated and 42 were downregulated in collagen $(+)$ E. faecalis in comparison to collagen(-) E. faecalis (Tables 1 and 2, respectively). Genes for ABC transporter permease and dipeptide epimerase showed the highest log2FC (fold change) of $5.97(P=0.001)$. The upregulated DEGs included multiple genes for sugar uptake, peptide and amino acid metabolism, and membrane structural proteins. Three virulence-associated genes encoding a matrixin family metalloprotease, WxL domain-containing protein, and LPXTG cell wall anchor domain-containing protein were also included in the upregulated DEGs. The downregulated DEGs included genes associated with membrane structure (LysM peptidoglycan-binding domain-containing protein) and phage.

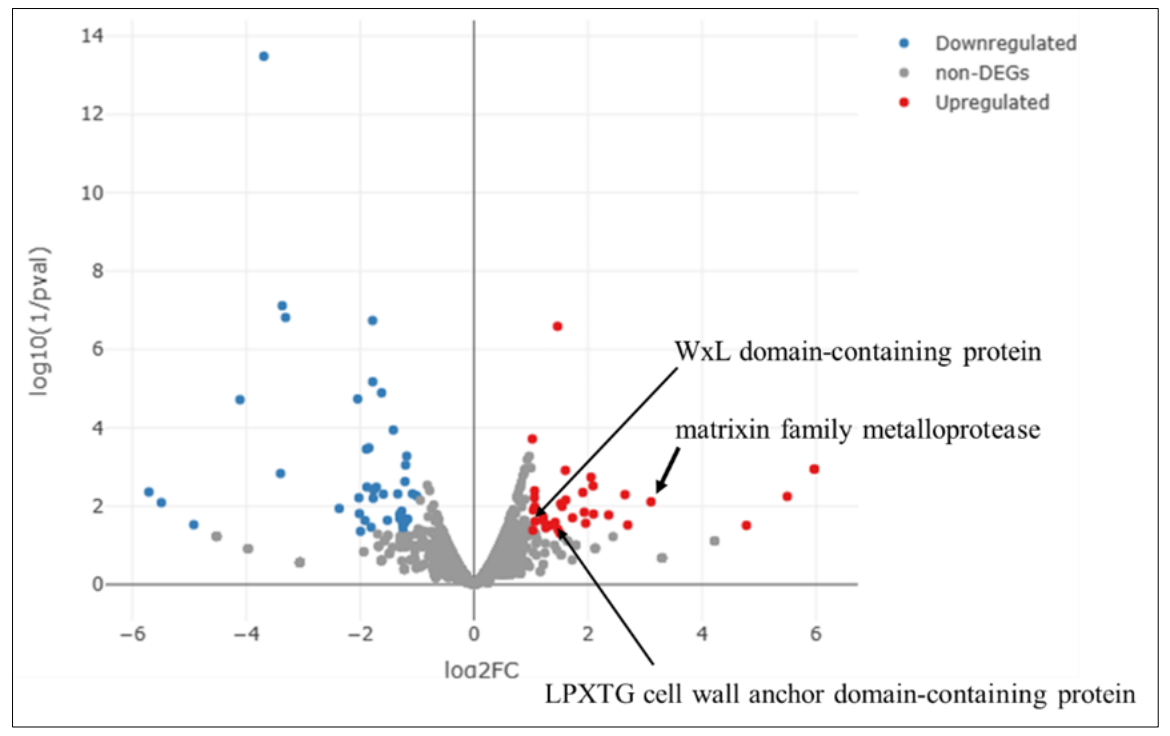

Figure 1 Volcano plot of gene distribution in collagen(+) E. faecalis compared with collagen(-) E. faecalis samples. The locations of the three upregulated differentially expressed genes (DEGs) associated with the virulence are indicated by arrows

Table 1 DEGs (upregulated)

\begin{tabular}{|l|l|c|l|}
\hline Gene ID & Description & logFC & $\boldsymbol{P}$-value \\
\hline WMC_RS14520 & beta-glucoside-specific PTS transporter subunit IIABC & 1.46 & $\begin{array}{l}2.57 \mathrm{E}- \\
07\end{array}$ \\
\hline WMC_RS14735 & glycerate kinase & 1.02 & 0.00019 \\
\hline WMC_RS08950 & ABC transporter permease & 5.97 & 0.00113 \\
\hline WMC_RS10065 & dipeptide epimerase & 5.97 & 0.00113 \\
\hline WMC_RS03480 & SDR family oxidoreductase & 1.60 & 0.00121 \\
\hline WMC_RS05280 & $\begin{array}{l}\text { bifunctional 4-hydroxy-2-oxoglutarate aldolase/2-dehydro-3-deoxy- } \\
\text { phosphogluconate aldolase }\end{array}$ & 2.05 & 0.00182 \\
\hline
\end{tabular}




\begin{tabular}{|l|l|l|l|}
\hline WMC_RS03470 & transcriptional regulator GutM & 2.09 & 0.00304 \\
\hline WMC_RS15835 & hypothetical protein & 1.06 & 0.00403 \\
\hline WMC_RS16230 & PTS sugar transporter subunit IIC & 1.91 & 0.00445 \\
\hline WMC_RS07700 & matrixin family metalloprotease & 2.65 & 0.00508 \\
\hline WMC_RS14035 & helix-hairpin-helix domain-containing protein & 5.50 & 0.00563 \\
\hline WMC_RS17445 & putative holin-like toxin & 1.06 & 0.00604 \\
\hline WMC_RS08470 & transporter substrate-binding domain-containing protein & 1.61 & 0.00694 \\
\hline WMC_RS09810 & NAD(P)H-dependent oxidoreductase subunit E & 3.10 & 0.00773 \\
\hline WMC_RS10680 & ethanolamine ammonia-lyase subunit EutB & 1.52 & 0.00866 \\
\hline WMC_RS09815 & NADH-quinone oxidoreductase subunit F & 1.54 & 0.01015 \\
\hline WMC_RS13675 & PTS sugar transporter subunit IIB & 1.07 & 0.01030 \\
\hline WMC_RS11820 & DUF87 domain-containing protein & 1.04 & 0.01260 \\
\hline WMC_RS13940 & purine permease & 1.93 & 0.01425 \\
\hline WMC_RS09300 & hypothetical protein & 2.09 & 0.01591 \\
\hline WMC_RS08515 & PTS sugar transporter subunit IIB & 2.36 & 0.01681 \\
\hline WMC_RS17350 & PTS system mannose/fructose/sorbose family transporter subunit IID & 1.21 & 0.01915 \\
\hline WMC_RS06620 & LPXTG cell wall anchor domain-containing protein & 1.72 & 0.01980 \\
\hline WMC_RS10100 & PTS fructose transporter subunit IIB & 1.21 & 0.02284 \\
\hline WMC_RS17220 & WxL domain-containing protein & 1.07 & 0.02450 \\
\hline WMC_RS03725 & pyridoxal phosphate-dependent aminotransferase & 1.42 & 0.02597 \\
\hline WMC_RS16235 & PTS sugar transporter subunit IIB & 2.00 & 0.02723 \\
\hline WMC_RS13605 & IS6-like element IS1216 family transposase & 1.26 & 0.02924 \\
\hline WMC_RS10640 & TIGR02536 family ethanolamine utilization protein & 2.70 & 0.03019 \\
\hline WMC_RS17550 & cytosine permease & 1.33 & 0.03083 \\
\hline WMC_RS11745 & bifunctional lysozyme/C40 family peptidase & 4.78 & 0.03096 \\
\hline WMC_RS08475 & amino acid ABC transporter ATP-binding protein & 1.26 & 0.03576 \\
\hline WMC_RS18065 & IS6-like element IS1216 family transposase & 1.47 & 0.03933 \\
\hline WMC_RS03465 & PTS glucitol/sorbitol transporter subunit IIC & 1.03 & 0.04103 \\
\hline WMC_RS10625 & ethanolamine utilization protein EutQ & 1.51 & 0.04906 \\
\hline & & & \\
\hline
\end{tabular}

Table 2 DEGs (downregulated)

\begin{tabular}{|l|l|c|c|}
\hline Gene ID & Description & logFC & $P$-value \\
\hline WMC_RS16775 & putative holin-like toxin & -3.68 & $3.30 \mathrm{E}-14$ \\
\hline WMC_RS12355 & HK97 gp10 family phage protein & -3.36 & $7.67 \mathrm{E}-08$ \\
\hline WMC_RS12365 & hypothetical protein & -3.31 & $1.53 \mathrm{E}-07$ \\
\hline WMC_RS12335 & tape measure protein & -1.78 & $1.83 \mathrm{E}-07$ \\
\hline WMC_RS12400 & phage portal protein & -1.78 & $6.66 \mathrm{E}-06$ \\
\hline WMC_RS12345 & Ig-like domain-containing protein & -1.62 & $1.28 \mathrm{E}-05$ \\
\hline WMC_RS12405 & PBSX family phage terminase large subunit & -2.04 & $1.84 \mathrm{E}-05$ \\
\hline WMC_RS13175 & ParB N-terminal domain-containing protein & -4.11 & $1.92 \mathrm{E}-05$ \\
\hline WMC_RS12375 & DUF5309 domain-containing protein & -1.42 & 0.00011 \\
\hline WMC_RS12340 & hypothetical protein & -1.85 & 0.00032 \\
\hline WMC_RS13245 & hypothetical protein & -1.89 & 0.00035 \\
\hline WMC_RS12325 & phage tail protein & -1.18 & 0.00052 \\
\hline
\end{tabular}




\begin{tabular}{|l|l|l|l|}
\hline WMC_RS12280 & LysM peptidoglycan-binding domain-containing protein & -1.20 & 0.00089 \\
\hline WMC_RS06650 & hypothetical protein & -3.40 & 0.00146 \\
\hline WMC_RS12380 & DUF4355 domain-containing protein & -1.21 & 0.00234 \\
\hline WMC_RS12385 & hypothetical protein & -1.88 & 0.00324 \\
\hline WMC_RS17480 & NusG domain II-containing protein & -1.72 & 0.00327 \\
\hline WMC_RS12350 & hypothetical protein & -1.76 & 0.00384 \\
\hline WMC_RS12285 & phage holin & -5.71 & 0.00434 \\
\hline WMC_RS11580 & GHKL domain-containing protein & -1.08 & 0.00483 \\
\hline WMC_RS13715 & hypothetical protein & -1.34 & 0.00486 \\
\hline WMC_RS05935 & YibE/F family protein & -1.59 & 0.00492 \\
\hline WMC_RS11700 & response regulator transcription factor & -1.01 & 0.00544 \\
\hline WMC_RS04645 & 16S ribosomal RNA & -2.02 & 0.00611 \\
\hline WMC_RS09135 & hypothetical protein & -1.77 & 0.00627 \\
\hline WMC_RS16180 & hypothetical protein & -5.49 & 0.00811 \\
\hline WMC_RS13645 & transposase & -2.37 & 0.01147 \\
\hline WMC_RS11765 & hypothetical protein & -1.27 & 0.01340 \\
\hline WMC_RS12505 & YegP family protein & -2.01 & 0.01551 \\
\hline WMC_RS12475 & YqaJ viral recombinase family protein & -1.31 & 0.01619 \\
\hline WMC_RS17960 & DDE-type integrase/transposase/recombinase & -1.31 & 0.02036 \\
\hline WMC_RS14685 & hypothetical protein & -1.19 & 0.02132 \\
\hline WMC_RS12410 & small subunit of terminase & -1.16 & 0.02144 \\
\hline WMC_RS05030 & DUF4822 domain-containing protein & -1.52 & 0.02295 \\
\hline WMC_RS11740 & conjugal transfer protein & -1.91 & 0.02320 \\
\hline WMC_RS16000 & hypothetical protein & -1.26 & 0.02638 \\
\hline WMC_RS11975 & 5-bromo-4-chloroindolyl phosphate hydrolysis family protein & -1.22 & 0.02665 \\
\hline WMC_RS08075 & 23S ribosomal RNA & -4.92 & 0.02973 \\
\hline WMC_RS13725 & zinc ribbon domain-containing protein & -1.25 & 0.03393 \\
\hline WMC_RS12305 & hypothetical protein & -1.81 & 0.03461 \\
\hline WMC_RS04265 & ABC transporter ATP-binding protein & -1.99 & 0.04378 \\
\hline WMC_RS06395 & DUF3290 domain-containing protein & -1.24 & 0.04431 \\
\hline & DEGs, differentially expressed genes;logFC, log2(fold change). & & \\
\hline
\end{tabular}

Table 3 GO enrichment analysis and functional annotation of DEGs (upregulated)

\begin{tabular}{|c|c|c|c|c|}
\hline Category & GO.ID & \multicolumn{2}{|l|}{ Term (DEGs/annotated gene numbers) } & $P$-value \\
\hline $\mathrm{BP}$ & GO:0032196 & \multicolumn{2}{|l|}{ Transposition $(2 / 8)$} & 0.0052 \\
\hline \multirow[t]{2}{*}{$\mathrm{BP}$} & GO:0015074 & \multicolumn{2}{|l|}{ DNA integration $(2 / 18)$} & 0.026 \\
\hline & Gene ID & Description & $\operatorname{logFC}$ & $P$-value \\
\hline \multirow[t]{2}{*}{ DEGs } & WMC_RS13605 & IS6-like element IS1216 family transposase & 1.26 & 0.029 \\
\hline & WMC_RS18065 & IS6-like element IS1216 family transposase & 1.47 & 0.039 \\
\hline Category & GO.ID & \multicolumn{2}{|l|}{ Term (DEGs/annotated gene numbers) } & $P$-value \\
\hline $\mathrm{CC}$ & GO:0031012 & \multicolumn{2}{|l|}{ extracellular matrix $(1 / 1)$} & 0.020 \\
\hline $\mathrm{CC}$ & GO:0044421 & \multicolumn{2}{|l|}{ extracellular region part (1/1) } & 0.020 \\
\hline MF & GO:0004222 & \multicolumn{2}{|l|}{ metalloendopeptidase activity } & 0.034 \\
\hline \multirow{2}{*}{ DEG } & Gene ID & Description & $\log \mathrm{FC}$ & $P$-value \\
\hline & WMC_RS07700 & matrixin family metalloprotease & 2.65 & 0.0051 \\
\hline
\end{tabular}




\begin{tabular}{|l|l|l|l|l|}
\hline Category & GO.ID & \multicolumn{2}{|l|}{ Term (DEGs/annotated gene numbers) } & $P$-value \\
\hline MF & G0:0051537 & 2 iron, 2 sulfur cluster binding (1/1) & 0.017 \\
\hline \multirow{2}{*}{ DEG } & Gene ID & Description & logFC & $P$-value \\
\cline { 2 - 5 } & WMC_RS09810 & $\begin{array}{l}\text { NAD(P)H-dependent } \\
\text { subunit E }\end{array}$ & 3.10 & 0.0077 \\
\hline Category & GO.ID & Term (DEGs/annotated gene numbers) & $P$-value \\
\hline MF & GO:0005353 & fructose transmembrane transporter activity (1/2) & 0.034 \\
\hline \multirow{2}{*}{ MF } & G0:0015145 & $\begin{array}{l}\text { monosaccharide transmembrane transporter activity } \\
(1 / 2)\end{array}$ & 0.034 \\
\hline MF & G0:0015149 & hexose transmembrane transporter activity (1/2) & 0.034 \\
\hline \multirow{2}{*}{ MF } & G0:0022877 & $\begin{array}{l}\text { protein-N(PI)-phosphohistidine-fructose } \\
\text { phosphotransferase system transporter activity (1/2) }\end{array}$ & 0.034 \\
\hline MF & G0:0051119 & sugar transmembrane transporter activity (1/2) & 0.034 \\
\hline \multirow{2}{*}{ DEG } & Gene ID & Description & logFC & $P$-value \\
\cline { 2 - 5 } & WMC_RS10100 & PTS fructose transporter subunit IIB & 1.21 & 0.023 \\
\hline
\end{tabular}

GO, gene ontology; DEG, differentially expressed gene; BP, biological process; CC, cellular component; MF, molecular function; $\log F \mathrm{C}$, $\log 2$ (fold change).

Eleven GO terms, including metalloendopeptidase activity (GO:0004222) and two related GO terms (GO:0031012, GO:0044421), were significantly enriched in the set of upregulated DEGs (Table 3). Sugar transmembrane transporter activity (GO:0051119) and four related GO terms (GO:0005353, GO:0015145, GO:0015149, G0:0022877), transposition (GO:0032196), DNA integration (GO:0015074), and 2 iron, 2 sulfur cluster binding (GO:0051537) were also enriched. The oriented graph of the category MF is shown as a representative in Fig. 2. In this category, metalloendopeptidase activity, 2 iron, 2 sulfur cluster binding, and five sugar transport-related GO terms were enriched.

Four GO terms, aminoglycan catabolic process (GO:0006026), glycosaminoglycan catabolic process (G0:0006027), lysozyme activity (GO:0003796), and peptidoglycan muralytic activity (G0:0061783) were significantly enriched in the set of downregulated DEGs (Table 4).

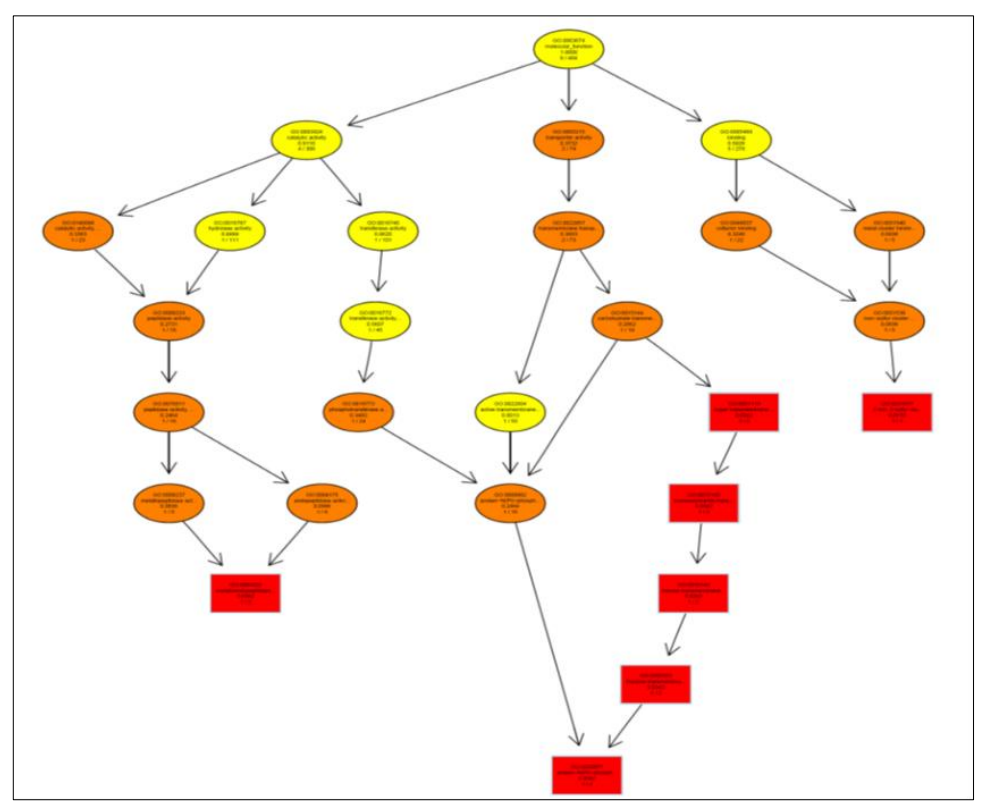

Figure 2 Directed acyclic graph of GO enrichment analysis of category MF (molecular function) and DEG functional annotation. Rectangles represent significantly enriched GO terms, including GO ID, $P$-value, and the numbers of DEGs/annotated gene numbers. The upstream GO function includes the downstream GO function (arrows) and the color intensity represents the degree of GO function enrichment 
Table 4 GO enrichment analysis and functional annotation of DEGs (downregulated)

\begin{tabular}{|l|l|l|l|l|}
\hline Category & \multicolumn{1}{|c|}{ GO.ID } & \multicolumn{1}{|c|}{ Term (DEGs/annotated gene numbers) } & $P$-value \\
\hline BP & GO:0006026 & aminoglycan catabolic process (1/1) & 0.0062 \\
\hline BP & GO:0006027 & glycosaminoglycan catabolic process (1/1) & 0.0062 \\
\hline MF & GO:0003796 & lysozyme activity (1/1) & 0.0043 \\
\hline MF & GO:0061783 & peptidoglycan muralytic activity (1/1) & 0.0043 \\
\hline \multirow{2}{*}{ DEG } & Gene ID & Description & logFC & -value \\
\cline { 2 - 5 } & WMC_RS12280 & $\begin{array}{l}\text { LysM peptidoglycan-binding domain- } \\
\text { containing protein }\end{array}$ & -1.20 & 0.00089 \\
\hline
\end{tabular}

\subsection{MMP activity}

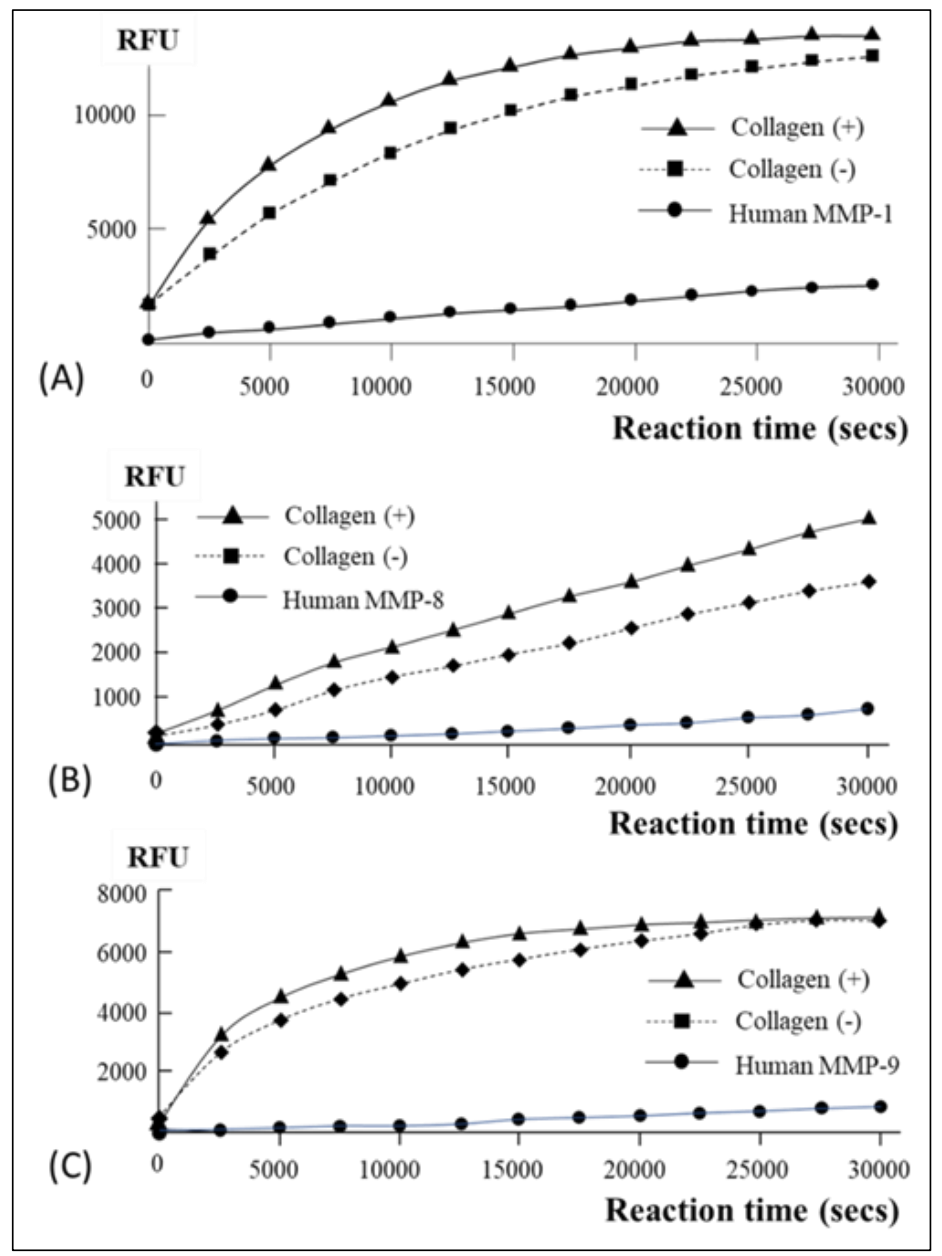

Figure 3 Kinetics of generic MMP activity (A), MMP-8 activity (B), and MMP-9 activity (C) of collagen(+) and collagen(-) E. faecalis. RFU denotes the fluorescence intensity resulting from degradation of the substrates and is shown after normalization relative to the negative control. Recombinant human MMP-1, MMP-8, and MMP-9 were used as positive controls

The results of DEG and GO enrichment analysis identified a matrixin family metalloprotease gene that may upregulate MMP activity in E. faecalis in contact with type I collagen. Based on the results of transcriptome analysis, phenotypic alterations of $E$. faecalis in MMP activity were examined. 


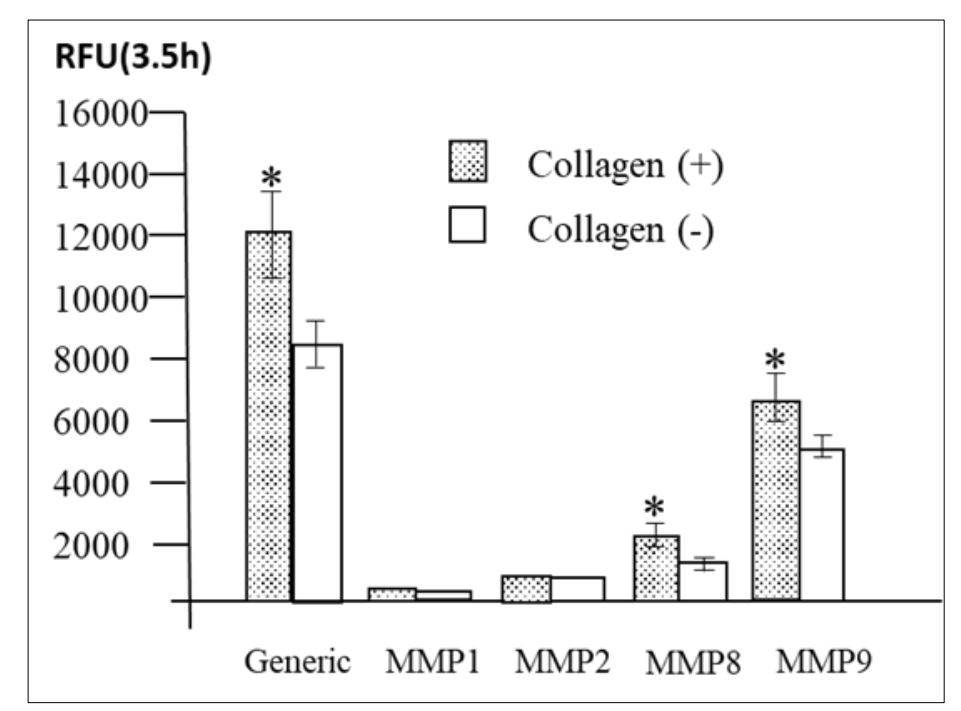

Figure 4 MMP activities of collagen( + ) and collagen(-) E. faecalis at $3.5 \mathrm{~h}$. Triplicate measurements were performed twice, and the data are shown as the mean $\pm \mathrm{SD}$. ${ }^{*} \mathrm{P}<0.05$, significant differences in generic MMP, MMP-8, and MMP-9 activities between collagen(+) and collagen(-) E. faecalis (Mann-Whitney U test). RFU denotes the fluorescence intensity resulting from degradation of the substrates

Generic MMP, MMP-1, 2, 8, and MMP-9 activities of the bacterial cell lysate were examined and were compared between collagen(+) and collagen(-) E. faecalis. Representative kinetics curves of generic MMP, MMP-8, and MMP-9 activities of the bacterial cells are shown in Fig. 3A - C. The curves of generic MMP and MMP-9 activities reached a plateau at the end-point of the measurements, while that of MMP-8 activity did not reach a plateau at the end-point. The rates of increase in RFU resulting from generic MMP, MMP-8, and MMP-9 activities of collagen(+) E. faecalis were higher than those of collagen(-) E. faecalis. There were no differences in MMP-1 or MMP-2 activities between the two different culture conditions (data not shown).

The RFU at $3.5 \mathrm{~h}$ were compared between collagen(+) and collagen(-) E. faecalis (Fig. 4). The RFU resulting from generic MMP, MMP-8, and MMP-9 activities of the collagen(+) E. faecalis were significantly higher than those of collagen(-) E. faecalis $(P<0.05)$.

\section{Discussion}

E. faecalis, a common commensal of the gastrointestinal tract of humans and other animals, has emerged as an important opportunistic pathogen that causes a wide variety of systemic diseases [1,2]. The bacterium has also been implicated in the pathogenesis of root canal infection, especially in persistent periapical periodontitis [3]. E. faecalis can adhere to the root canal walls, invade into the dentinal tubules, and form a biofilm that makes this bacterium resistant to disinfectants [12]. For bacteria to act as pathogens, they first need to adhere and colonize the host tissue. As E. faecalis can colonize both systemic organs and dentin, collagen is the possible host target of this bacterium. Among the collagens, type I collagen is the key molecule in endodontic infections, and a number of studies have investigated the underlying pathogenic mechanisms [12 - 14]. To our knowledge, however, there have been no reports of comprehensive gene expression analyses of $E$. faecalis induced by type I collagen. Our goal was to identify common virulence factors of $E$. faecalis between systematic disease and dentin infections for the prevention of these diseases. As the first step, transcriptome analysis of $E$. faecalis cultivated with type I collagen was performed.

The upregulated genes included three virulence-associated genes (LPXTG cell wall anchor domain-containing protein, WxL domain-containing protein, and matrixin family metalloprotease). The LPXTG cell anchor domain is conserved among the virulence proteins of gram-positive cocci. A collagen-binding protein of Staphylococcus aureus, Can, contains the same domain [15]. In addition, Cnm, a collagen-binding protein of Streptococcus mutans, contains the LPXPTG domain [16]. Ace, an adhesin with an LPXPTG domain, has been reported to be a collagen-binding protein in E. faecalis [17]. These adhesins have been reported to be closely related to the virulence of these bacteria [14 - 17]. The functions of the LPXTG cell wall anchor domain-containing protein identified in this study are still unclear, and further studies are required to elucidate its functions. The WxL domain-containing protein has also been reported to bind to type I collagen and fibronectin in Enterococcus faecium [18,19]. E. faecalis and E. faecium are closely related species, and the WxL domain-containing protein identified here probably has the same function. Although Ace is now recognized as a central 
virulence adhesin in E. faecalis, further studies of the WxL domain-containing protein are required. The Ace was not upregulated in this study.

In GO enrichment analysis, the GO term metalloendopeptidase activity (G0:0004222) was enriched, and the matrixin family metalloprotease gene identified as an upregulated DEG was also suggested to be a putative virulence factor of $E$. faecalis. This molecule consists of 185 amino acids, and contains a zinc-binding domain. The genome database of $E$. faecalis ATCC 19433 includes six metalloprotease genes and another six metallopeptidase genes. The results of the present study suggested that the identified matrixin family metalloprotease may be a key molecule for elucidating the virulence associated with type I collagen among these proteases. The functions of this molecule have not been reported. Although the details are unclear, the results of MMP assay in this study suggested that the matrixin family metalloprotease may have MMP-8- or MMP-9-like activity. Although gelatinase (GelE) of E. faecalis [20] has been reported to be a major protease that acts as a virulence factor, this gene was not upregulated in this study.

The results of GO enrichment analysis and the list of upregulated DEGs suggested the alteration of nutrient uptake of $E$. faecalis through contact with type I collagen. Among the 35 upregulated DEGs, 15 were related to sugar transport or metabolism.

GutM is a DNA-binding transcription factor that regulates an operon involved in transport and utilization of glucitol [21], and permease and aldolase genes were also included in the upregulated DEGs. In addition, three genes for amino acid and peptide metabolism were included in the upregulated DEGs (pyridoxal phosphate-dependent aminotransferase, amino acid ABC transporter ATP-binding protein, and bifunctional lysozyme/C40 family peptidase) [22]. It has been reported that gene expression in E. faecalis is regulated to ensure cell survival under altered environmental conditions [23]. The upregulated DEGs associated with nutrient uptake and metabolism identified here may play important roles in the adaptation and survival of E. faecalis in host tissue with type I collagen.

The downregulated enriched GO terms and DEG may be related to the membrane structure. LysM modules recognize polysaccharides containing $N$-acetylglucosamine residues, including peptidoglycan, an essential component of the bacterial cell wall [24]. However, the molecular mechanisms underlying LysM-peptidoglycan interactions and their functions remain unclear. Five phage-related genes were included in the downregulated DEGs for unknown reasons.

Our attention was focused on the enriched G0 term, metalloendopeptidase activity, and the phenotypic alterations of MMP activity of the cells reacting to type I collagen were examined. In the present study, generic MMP, MMP-8, and MMP-9 activities were enhanced in E. faecalis exposed to type I collagen. The results suggested that these MMP activities of E. faecalis may play important roles in colonization and virulence in host tissue containing type I collagen. The matrixin family metalloprotease may be a key molecule for enhanced activity. Marashdeh et al. [25] reported that the MMP activities of $E$. faecalis showed different activity profiles among the strains, but the greatest activity was seen for MMP-8 or MMP-9, consistent with our results. They also reported that MMP activities of E. faecalis were 50 times higher than those of dentinal and salivary enzymes. MMP-8 is a collagenase that cleaves native triple-helical type I, II, and III collagens [26], and MMP-9 is a gelatinase that can degrade collagens [27]. These types of enzymes of E. faecalis may play important roles in degradation of dentinal collagen rather than salivary or dentinal enzymes, and may be common virulence factors between dentin and systemic infections.

\section{Conclusion}

A set of genes in E. faecalis induced by type I collagen were identified. The gene for matrixin family metalloprotease, LPXTG cell wall anchor domain-containing protein, WxL domain-containing protein, and a series of genes for energy uptake were upregulated. These molecules may be involved in the pathogenesis of dentin infection. MMP-8 and MMP-9 activities of $E$. faecalis were enhanced by stimulation with type I collagen, suggesting that they play enzymatic roles in dentin degradation.

\section{Compliance with ethical standards}

\section{Acknowledgments}

This study was supported in part by a Grant-in-Aid for Scientific Research (Grant number: 18K09613) from the Japan Society for the Promotion of Science. 


\section{Disclosure of conflict of interest}

The authors have no conflicts of interest to declare.

\section{References}

[1] Raza T, Ullah SR, Mehmood K, Andleeb S. Vancomycin resistant Enterococci: A brief review. J Pak Med Assoc. 2018; 68: 768-772.

[2] Rice LB. Emergence of vancomycin-resistant enterococci. Emerg Infect Dis. 2001; 7: 183-187.

[3] Sedgley C, Buck G, Appelbe O. Prevalence of Enterococcus faecalis at multiple oral sites in endodontic patients using culture and PCR. J Endod. 2006; 32: 104-109.

[4] Salah R, Dar-Odeh N, Abu Hammad O, Shehabi AA. Prevalence of putative virulence factors and antimicrobial susceptibility of Enterococcus faecalis isolates from patients with dental Diseases. BMC Oral Health. 2008; 8: 17.

[5] Zhang C, Du J, Peng Z. Correlation between Enterococcus faecalis and Persistent Intraradicular Infection Compared with Primary Intraradicular Infection: A Systematic Review. J Endod. 2015; 41: 1207-1213.

[6] Sundqvist G, Figdor D, Persson S, Sjögren U. Microbiologic analysis of teeth with failed endodontic treatment and the outcome of conservative re-treatment. Oral Surg Oral Med Oral Pathol Oral Radiol Endod. 1998; 85: 86-93.

[7] Orstavik D, Haapasalo M. Disinfection by endodontic irrigants and dressings of experimentally infected dentinal tubules. Endod Dent Traumatol. 1990; 6: 142-9.

[8] Fabricius L, Dahlén G, Holm SE, Möller AJ. Influence of combinations of oral bacteria on periapical tissues of monkeys. Scand J Dent Res. 1982; 90: 200-2006.

[9] Sood S, Malhotra M, Das BK, Kapil A. Enterococcal infections \& antimicrobial resistance. Indian J Med Res. 2008; 128: $111-121$.

[10] Kayaoglu G, Ørstavik D. Virulence factors of Enterococcus faecalis: relationship to endodontic disease. Crit Rev Oral Biol Med. 2004; 15: 308-320.

[11] Kim D, Paggi JM, Park C, Bennett C, Salzberg SL. Graph-based genome alignment and genotyping with HISAT2 and HISAT-genotype. Nat Biotechnol. 2019; 37: 907-915.

[12] Kayaoglu G, Erten H, Ørstavik D. Possible role of the adhesin ace and collagen adherence in conveying resistance to disinfectants on Enterococcus faecalis. Oral Microbiol Immunol. 2008; 23: 449-454.

[13] Love RM. Enterococcus faecalis--a mechanism for its role in endodontic failure. Int Endod J. 2001; 34: $399-405$.

[14] Hubble TS, Hatton JF, Nallapareddy SR, Murray BE, Gillespie MJ. Influence of Enterococcus faecalis proteases and the collagen-binding protein, Ace, on adhesion to dentin. Oral Microbiol Immunol. 2003; 18: 121-126.

[15] Gillaspy AF, Patti JM, Pratt FL Jr, Iandolo JJ, Smeltzer MS. The Staphylococcus aureus collagen adhesin-encoding gene (cna) is within a discrete genetic element. Gene. 1997; 196: 239-248.

[16] Nomura R, Ogaya Y, Nakano K. Contribution of the Collagen-Binding Proteins of Streptococcus mutans to Bacterial Colonization of Inflamed Dental Pulp. PLoS One. 2016; 11(7): e0159613.

[17] Rich RL, Kreikemeyer B, Owens RT, LaBrenz S, Narayana SV, Weinstock GM, Murray BE, Höök M. Ace is a collagenbinding MSCRAMM from Enterococcus faecalis. J Biol Chem. 1999; 274: 26939-26945.

[18] Galloway-Peña JR, Liang X, Singh KV, Yadav P, Chang C, La Rosa SL, Shelburne S, Ton-That H, Höök M, Murray BE. The identification and functional characterization of WxL proteins from Enterococcus faecium reveal surface proteins involved in extracellular matrix interactions. J Bacteriol. 2015; 197: 882-892.

[19] Brinster S, Posteraro B, Bierne H, Alberti A, Makhzami S, Sanguinetti M, Serror P. Enterococcal leucine-rich repeat-containing protein involved in virulence and host inflammatory response. Infect Immun. 2007; 75: 44634471.

[20] Wang L, Dong M, Zheng J, Song Q, Yin W, Li J, Niu W. Relationship of biofilm formation and gelE gene expression in Enterococcus faecalis recovered from root canals in patients requiring endodontic retreatment. J Endod. 2011; 37: 631-636.

[21] Yamada M, Saier MH Jr. Positive and negative regulators for glucitol (gut) operon expression in Escherichia coli. J Mol Biol. 1988; 203: 569-583. 
[22] Nemoto TK, Ohara Nemoto Y. Dipeptidyl-peptidases: Key enzymes producing entry forms of extracellular proteins in asaccharolytic periodontopathic bacterium Porphyromonas gingivalis. Mol Oral Microbiol. 2021; 36: 145-156.

[23] Giard JC, Hartke A, Flahaut S, Boutibonnes P, Auffray Y. Glucose starvation response in Enterococcus faecalis JH22: survival and protein analysis. Res Microbiol. 1997; 148: 27-35.

[24] Mesnage S, Dellarole M, Baxter NJ, Rouget JB, Dimitrov JD, Wang N, Fujimoto Y, Hounslow AM, Lacroix-Desmazes S, Fukase K, Foster SJ, Williamson MP. Molecular basis for bacterial peptidoglycan recognition by LysM domains. Nat Commun. 2014; 5: 4269.

[25] Marashdeh MQ, Gitalis R, Lévesque C, Finer Y. Endodontic pathogens possess collagenolytic properties that degrade human dentine collagen matrix. Int Endod J. 2019; 52: 416-423.

[26] Perumal S, Antipova 0, Orgel JP. Collagen fibril architecture, domain organization, and triple-helical conformation govern its proteolysis. Proc Natl Acad Sci U S A. 2008; 105: 2824-2829.

[27] Hannas AR, Pereira JC, Granjeiro JM, Tjäderhane L. The role of matrix metalloproteinases in the oral environment. Acta Odontol Scand. 2007; 65: 1-13. 